\title{
Just the facts: management of electrical storm and recurrent ICD shocks in the emergency department
}

\author{
Lindsay Cheskes ${ }^{1} \cdot$ Ashley Krywenky $^{1} \cdot$ Mouhannad M. Sadek $^{2}$ \\ Received: 12 June 2020 / Accepted: 16 November 2020 / Published online: 18 January 2021 \\ (c) Canadian Association of Emergency Physicians (CAEP)/ Association Canadienne de Médecine d'Urgence (ACMU) 2021
}

Keywords Emergency medicine $\cdot$ Resuscitation $\cdot$ Cardiac disease $\cdot$ Arrhythmia

\section{Case}

A 65-year-old male with ischemic cardiomyopathy, secondary prevention implantable cardioverter-defibrillator (ICD), diabetes, dyslipidemia and hypertension presents to your emergency department (ED). He reports 10 ICD shocks over the past $12 \mathrm{~h}$. He reports palpitations and light-headedness preceding the ICD shocks. There was no loss of consciousness, chest pain, nor shortness of breath. His vital signs are stable and he is afebrile. His exam is unremarkable.

\section{Clinical questions}

\section{What is the definition and clinical epidemiology of electrical storm?}

Electrical storm refers to the occurrence of three or more episodes of sustained ventricular tachycardia (VT) or ventricular fibrillation (VF) over a 24-h period. In patients with an ICD, the definition of electrical storm is accepted as three appropriate detections of ventricular arrhythmia that lead to ICD therapies (either anti-tachycardia pacing or shock) within $24 \mathrm{~h}$. The incidence of electrical storm after ICD implantation ranges from $4 \%$ (primary prevention ICDs) [1] to $33.1 \%$ (secondary prevention ICDs) [2]. It is associated with high morbidity and mortality, with studies reporting a 5 - to 18 -fold increase in mortality in the first 3 months after

Ashley Krywenky

akrywenky@toh.ca

1 Department of Emergency Medicine, The Ottawa Hospital, University of Ottawa, 1053 Carling Ave, Ottawa, ON K1Y4E9, Canada

2 Division of Cardiology, University of Ottawa Heart Institute, Ottawa, ON, Canada diagnosis [1]. Monomorphic VT is by far the most common dysrhythmia encountered in electrical storm.

\section{What are the underlying causes of electrical storm?}

The causes of electrical storm include scar-mediated reentry due to myocardial injury including prior myocardial infarction, acute ischemia, acute decompensated heart failure, electrolyte abnormalities, drug toxicities, sepsis, thyrotoxicosis and genetic conditions (ex. catecholaminergic polymorphic VT). While an effort should be made to identify and treat reversible causes, an inciting factor is not identified in up to $66 \%$ of cases [3].

\section{What are the clinical presentations of electrical storm in the ICD patient?}

Patients with an ICD who have electrical storm typically present with multiple ICD therapies. Symptoms can include pain from ICD discharges, palpitations, lightheadedness, syncope, or cardiac arrest. It is important to note that in patients in whom the VT rate is below the ICD therapy threshold (typically 170-200 beats per minute for primary prevention ICDs [4]), patients may present with monomorphic VT that is not detected or treated by the ICD. ICDs can be reprogrammed to detect these "slow" ventricular tachycardias, with the new detection rate set 10-20 beats per minute below the rate at which clinical VT occurred.

\section{What is the difference between appropriate and inappropriate ICD therapies?}

An appropriate ICD therapy refers to the ICD delivering a therapy for a malignant ventricular arrhythmia (VT or VF). An inappropriate ICD therapy is one delivered in the absence of a malignant ventricular arrhythmia. Inappropriate 
Fig. 1 Approach to assessment and management of recurrent ICD discharges in the ED (ICD implantable cardioverter defibrillator, $A C L S$ advanced cardiovascular life support, $V T$ ventricular tachycardia, pMVT polymorphic ventricular tachycardia, $m V T$ monomorphic ventricular tachycardia)

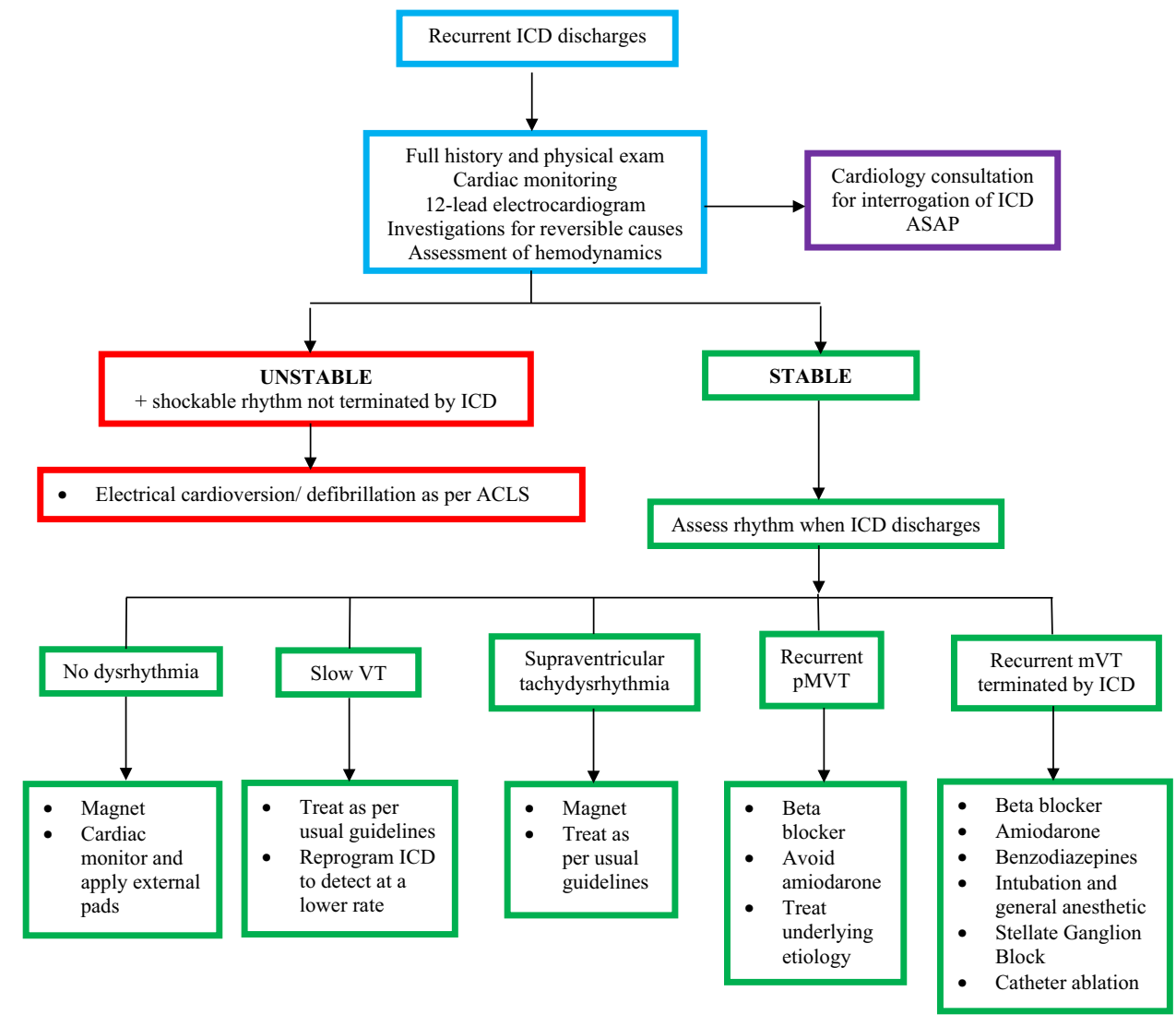

ICD therapies account for $30 \%$ of all ICD therapies [5] and may be due to non-malignant supraventricular arrhythmias (e.g. atrial fibrillation) or over-sensing. Causes of over-sensing include over-sensing of true cardiac electrical signals (such as T-wave sensing or double-sensing of very wide QRS complexes) and sensing of signals unrelated to cardiac electrophysiology (such as a fractured lead, skeletal myopotentials, or external electromagnetic interference) [5].

\section{What treatment should you initiate for this patient?}

Place the patient on cardiac monitors, initiate investigations for reversible causes, obtain an ECG and assess hemodynamics. For hemodynamically unstable patients with a shockable rhythm that is not terminated by the ICD, perform electrical cardioversion or defibrillation as per Advanced Cardiovascular Life Support (ACLS) guidelines. Position the pads $8-10 \mathrm{~cm}$ from the ICD to prevent ICD dysfunction. Following patient stabilization, prompt interrogation of the device should be undertaken.

If inappropriate therapies are suspected, apply a magnet over the ICD impulse generator. Application of a magnet disables tachycardia detection without interfering with bradycardia pacing functions. As a result, the patient will no longer receive shocks or anti-tachycardia pacing. While the magnet is in place, it is important that the patient remains on cardiac monitors with external pads applied in the event of a true ventricular arrhythmia (Fig. 1).

If a slow VT is present, the ICD need not be deactivated, and the VT is treated according to usual guidelines. If a supraventricular tachydysrhythmia is present, apply the magnet and treat the tachydysrhythmia according to usual guidelines.

For hemodynamically stable patients in whom the ICD has provided appropriate and successful therapy, treatment is based on the offending arrhythmia. In all patients, place external pads in case resuscitation is required, and have the ICD interrogated promptly.

Patients with monomorphic VT typically have scarrelated re-entry as the etiology. Attempts to identify and treat reversible triggers and to provide medical therapy to prevent further shocks should be undertaken. This includes oral beta blockers (e.g. propranolol $40 \mathrm{mg}$ PO q6h) as well as amiodarone (150 mg IV over $10 \mathrm{~min}$ followed by $1 \mathrm{mg} / \mathrm{min}$ for $6 \mathrm{~h}$, then $0.5 \mathrm{mg} / \mathrm{min}$ for $18 \mathrm{~h}$ ) as the preferred antiarrhythmic agent. If the patient continues to experience arrhythmias, benzodiazepines (ex. ativan 1-2 mg IV) should be given for further sympatholysis. If this fails, intubation and deep sedation with general anesthetic should be considered. Patients with polymorphic VT typically have either long-QT or coronary ischemia as the etiology, which should be addressed. In this setting, 
amiodarone administration is cautioned as it can exacerbate further arrhythmia.

In patients who continue to experience device therapies despite the above treatments, consider advanced therapies such as neuraxial modulation with a stellate ganglion block. Stellate ganglion block is a percutaneous nerve block procedure that blocks sympathetic outflow to the heart, and is a temporizing measure in refractory electrical storm until a definitive procedure is arranged. Catheter ablation has been shown to reduce future incidence of electrical storm [2]) and can be performed after patient stabilization.

\section{Key points}

- Electrical storm refers to the occurrence of three or more episodes of sustained VT or VF in a 24-h period. In the ICD patient, electrical storm is defined as three appropriate detections of ventricular arrhythmia that lead to ICD therapies in $24 \mathrm{~h}$.

- It is important to assess hemodynamics, the patient's presenting rhythm and whether ICD shocks are appropriate or inappropriate, as this influences management.

- A stepwise approach to electrical storm in the ICD patient includes oral beta blockers, amiodarone (in the absence of polymorphic VT), benzodiazepines, intuba- tion and general anesthetic, and consideration for stellate ganglion block and catheter ablation procedures.

\section{Compliance with ethical standards}

Conflict of interest The Authors declare no conflict of interest.

\section{References}

1. Sesselberg HW, Moss AJ, McNitt S, Zareba W, Daubert JP, Andrews ML, et al. Ventricular arrhythmia storms in postinfarction patients with implantable defibrillators for primary prevention indications: a MADIT-II substudy. Heart Rhythm. 2007;4(11):1395-402.

2. Sapp JL, Wells GA, Parkash R, Stevenson WG, Blier L, Sarrazin JF, et al. Ventricular tachycardia ablation versus escalation of antiarrhythmic drugs. N Engl J Med. 2016;375(2):111-21.

3. Grieco D, Borrelli A, de Ruvo E, Scara' A, Sciarra L, Della Bona $\mathrm{R}$, et al. Treatment of the arrhythmic storm. Eur Heart J Suppl. 2019;21(Suppl B):B23-4.

4. Moss AJ, Schuger C, Beck CA, Brown MW, Cannom DS, Daubert JP, et al. Reduction in inappropriate therapy and mortality through ICD programming. N Engl J Med. 2012;367(24):2275-83.

5. Iftikhar S, Mattu A, Brady W. ED evaluation and management of implantable cardiac defibrillator electrical shocks. Am J Emerg Med. 2016;34(6):1140-7. 